REVIEW ARTICLES

\title{
CRITICAL CARE MANAGEMENT OF HEAD INJURY - CURRENT CONCEPTS
}

\author{
Muhammad Qamarul Hoda* \\ Professor and Clinical Director ICU \\ Aga Khan University, Karachi, Pakistan \\ *Corresponding author E-mail : qamar.hoda@aku.edu
}

Key words: head injury, critical care, management, ICP versus CPP

Traumatic brain injury (TBI) is a leading cause of death and disability in children and adults in their most productive years of life. The damage to the brain occurs in two phases: primary and secondary. The primary brain injury results from the direct mechanical damage at the time of trauma. Secondary brain injury results due to neuronal damage from the systemic physiologic responses to the initial injury. The importance of hypotension and hypoxia as major causes of secondary injury has been recognized. Outcome from TBI can be improved by preventing or adequately treating these secondary insults timely.

In order to improve cerebral resuscitation, specific therapeutic goals and modalities have been advocated by different societies maintaining cerebral perfusion pressure (CPP), mean arterial pressure (MAP) and preventing or treating raised intra cranial pressure, mainly based on Monroe-Kellie doctrine, including CSF drainage, hyperventilation, mannitol, barbiturate induced coma, induced hypothermia and seizure prophylaxis.

The initial priorities in the management of TBI patient are same as with any trauma patient; establishment of an adequate airway, breathing and circulation. The cranial vault, a fixed space contains largely incompressible brain tissue, cerebrospinal fluid, blood and extracellular fluid ${ }^{1}$. Increase in the volume of one or the other within the intracranial compartment is poorly compensated and results in increase in intracranial pressure (ICP). This subsequently reduces cerebral perfusion pressure (CPP) and adversely affect outcome following head injury. Optimizing cerebral resuscitation at this stage can prevent or limit secondary brain injury. To achieve this, variations in the clinical management of TBI must be eliminated or reduced to a minimum. Guidelines regarding specific monitoring and therapeutic goals have been advocated by different socities ${ }^{2,3}$ and consensus conferences, and includes; ICP monitoring, maintenance of cerebral perfusion pressure (CPP), Mean Arterial Pressure (MAP)and preventing or treating raised ICP by CSF drainage, hyperventilation, use of mannitol, barbiturate induced coma, induced hypothermia and seizure prophylaxis.

\section{CRITICAL CARE MANAGEMENT:}

Once the TBI patient is stabilized and shifted to the ICU, the establishment of physiological monitoring facilitates and directs further management of these patients. Although there is no Class I evidence to support any of the specific interventions used in the management of head injuries ${ }^{4}$, it is generally agreed that the aim of intensive care of these patients is to prevent, identify and treat the causes of secondary brain injury namely; hypoxia, hypercapnia, hypotension, and hyperthermia.

Management of cerebral hemodynamics: To reduce secondary brain injury, avoidance of systemic hypotension is of paramount importance, as a single episode of hypotension increases the morbidity and mortality 
significantly ${ }^{5}$. Different approaches, targeting cerebral perfusion pressure and intra cranial pressure have been recommended for the management of TBI, with varying degree of success. Despite the fact that all these approaches have some physiologic basis, no one has proven superiority over any one approach. Although current guidelines by $\mathrm{BTF}^{2}$ emphasizes on the importance of maintaining adequate CPP, it is important to recognize that pathophysiology of TBI evolves over time, and treatment that is appropriate in the first few hours may not necessarily be optimal 2-3 days after injury.

The optimum level of CPP required following TBI has been subject to much debate. ${ }^{6,7}$ Aggressive fluid resuscitation and cardiovascular support with vasopressors and inotropes to augment MAP and maintain CPP $>70 \mathrm{mmHg}$ may reduce mortality and improves outcome by a reduction in cerebral ischemia. This strategy of increasing MAP and CPP may lead to high incidence of systemic complications, particularly Acute Respiratory Distress Syndrome $^{6}$. Based on this, BTF guidelines ${ }^{2}$ on target CPP has been revised downwards from 70 $\mathrm{mmHg}$ to $60 \mathrm{mmHg}$. However in selected patients, with evidence of regional or global ischemia, higher target may be appropriate.

ICP monitoring and management: Although no randomized controlled trials have been performed demonstrating that ICP monitoring improves outcome, ICP monitoring and aggressive management of raised ICP greater than $20 \mathrm{mmHg}$ has been recommended as level II recommendation, by Brain Trauma Foundation ${ }^{2}$ in all TBI patients with GCS 8 following non surgical resuscitation with abnormal CT scan. If CT scan is normal, monitoring is suggested (as level III) in patients above the age of forty years, with unilateral or bilateral motor posturing or those with extracranial trauma who remain hypotensive (systolic blood pressure less than 90 $\mathrm{mm} \mathrm{Hg}$ ) despite aggressive fluid resuscitation.

Currently available methods for ICP monitoring include extradural, subdural, intraparenchymal, and intraventricular catheters. Intraventricular catheters are preferred when possible, as these not only allow continuous measurements of ICP but also can be used of drainage of CSF to control raised ICP.
As does the maintenance of CPP, management of raised ICP greater than $20 \mathrm{mmHg}$ is also important ${ }^{2}$. Unfortunately existing trials have been too small to support or refute the existence of a real benefit from using CSF drainage, decompressive craniectomy, hyperventilation, mannitol, barbiturates, or corticosteroids, and induced hypothermia. ${ }^{4}$

Decompressive Craniectomy : Regarding surgical management of intractable intra-cranial hypertension, there is growing body of literature supporting the efficacy of decompressive craniectomy in reducing ICP, with improved brain oxygenation, especially after TBI., However in the absence of randomized, controlled trails, it is difficult to make a definitive judgment about its efficacy. ${ }^{10,11}$

Hyperventilation: The most potent non-surgical method to reduce raised ICP in acute phase of brain injury, should be considered as a temporizing measure, prior to urgent imaging or surgery in patients with unequivocal clinical signs of increased ICP or impending tentorial herniation (level III recommendation) ${ }^{2}$. Short term hyperventilation to a $\mathrm{PaCO}_{2}$ between 35$40 \mathrm{mmHg}$ is recommended. However based on current evidence use of empirical, excessive and prolonged hyperventilation should be avoided as this increases the risk of secondary ischemic changes. ${ }^{12}$ If hyperventilation is used, jugular venous oxygen saturation $\left(\mathrm{SjO}_{2}\right)$ or brain tissue oxygen saturation $\left(\mathrm{PbrO}_{2}\right)$ measurements are recommended to monitor oxygen delivery ${ }^{2}$.

Mannitol and other osmotherapeutic agents like hypertonic saline (HS) is an option similar to hyperventilation prior to imaging or evacuation in resuscitated patients with unequivocal clinical signs of increased ICP. Immediate reduction in ICP with mannitol $(0.25-1 \mathrm{~g} / \mathrm{kg})$, probably result from restoration of CPP and rheological changes in cerebral blood flow, rather than specific cerebral dehydration ${ }^{13}$. Empirical and regular use of mannitol is not recommended, as repeated doses may cause rebound increase in ICP especially in presence of damaged blood brain barrier.

Hypertonic saline: Hypertonic saline (HS) in concentration ranges from $3-7.5 \%$ has been tried 
in TBI patients. In addition to its osmotic effects, HS has hemodynamic, vasoregulatory, immunologic and neurochemical effects. Despite these beneficial effects current studies are inconclusive to clarify its role in head injury patients. $^{14}$

Induced hypothermia: Moderate hypothermia (32-340C) has been shown to decrease neuronal damage in animal and several other clinical studies. However, a systematic review of twelve randomized control trials by McIntyre and colleagues $^{15}$, of mild to moderate $\left(32^{0} \mathrm{C}-33^{0} \mathrm{C}\right)$ hypothermia following TBI noted only a small but significant decrease in the risk of death or poor neurological outcome. Despite this, the role of hypothermia in the management of TBI is still controversial and regarded as second tier option in patients with refractory intra cranial hypertension. ${ }^{16}$ However evidence suggests that rapid re-warming of patients who present with hypothermia may be deleterious and should be avoided.

With reference to barbiturate induced coma and use of steroids in head injury patients, despite strong experimental evidence, no definitive studies have shown any benefit from barbiturate coma in clinical trials.

The multinational MRC CRASH (Corticosteroid Randomization after Significant Head Injury) trial of more than ten thousands enrolled patients has concluded that the use of corticosteroid therapy was associated with increased acute mortality in head injury patients. $^{17}$

In summary, outcome from traumatic brain injury can be improved by preventing or adequately treating secondary insults responsible for decreasing cerebral perfusion. When CPP targeted approach is used it is important to recognize that pathophysiology of brain injury evolves over time, ranging from hypoperfusion in the first twenty four hours, hyperemic phase up to third day of injury followed by vasospastic phase with low-normal CBF.

\section{References:}

1. Monro A. Observations on the structure and function of the Nervous system. Edinburgh: Creech and Johnson, 1783.

2. Brain Injury Foundation, American Association of Neurological Surgeons, Joint Section on
Neurotrauma and Critical Care. Guidelines for the management of severe head injury. J Neurotrauma 2007;24: Supplement1.(www,braintrauma.org)

3. European Brain Injury Consortium (EBIC). EBIC-Guidelines for the management of severe head injury in adults. Acta Neurochir 1997;139:286-294

4. Roberts I, Schierhout G, Alderson P. Absence of evidence for the effectiveness of five interventions routinely used in the intensive care management of severe head injury: a systematic review. J Neurol Neurosurg Psychiatry 1998;65:729-33.

5. Chesnut RM, Marshall LF, Klauber MR. The role of secondary brain injury in determining outcome from severe head injury. J Trauma 1993;34:216222

6. Robertson CS, Valadka AB, Hannay HJ, et al. Prevention of secondary ischemic insults after severe head injury. Crit Care Med 1999;27:20862095

7. Steiner LA, Coles JP, Johnston AJ, et al. Response of posttraumatic pericontusional CBF and blood volume to an increase in CPP. J Cereb Blood Flow Mrtab 2003;23:1371-7.

8. Reithmeier T, Lohr M, Pakos P, Ketter G, Ernestus R-I. Relevance of $\mathrm{ICPand}^{\mathrm{PtiO}_{2}}$ for induction and timing of decompressive craniectomy in patients with malignant brain edema. Acta Neurochir (Wien) 2005;147:947-52

9. Stiefel MF, Heuer GG, Smith MJ, et al. Cerebral oxygenation following decompressive hemicraniectomy for the treatment of refractory intracranial hypertension. J Neurosurg 2004;101:241-7.

10. Morley NC, Berge E, Cruz-Flores S, Whittle IR. Surgical decompression for cerebral edema in acute ischemic attack. Cochrane Database Syst Rev 2002;CD003435.

11. Hutchinson PJ, Kirkpatrick PJ. Decompressive craniectomy in head injury. Curr Opin Crit Care 2004;10:101.

12. Cruz J. The first decade of continuous monitoring of jugular bulb oxyhemoglobin saturation: management strategies and clinical outcome. Crit Care Med 1998;26:344-351.

13. Muizelaar JP, Lutz HA, Becker DP. Effect of mannitol on ICP and CBF and correlation with pressure autoregulation in severely head injured patients J Neurosurg 1984;61:700-706.

14. Bhardwaj A, Ulatowski JA. Hypertonic saline solution in brain injury. Curr Opin Crit Care 2004;10:126-131

15. McIntyre LA, Fergusson DA, Herbert PC, et al. Prolonged therapeutic hypothermia after TBI in 
adults: a systematic review. JAMA 2003;289:2992-2999

16. Clifton GL, Miller ER, Choi SC et al. Lack of effect of induction of hypothermia after acute brain injury. N Engl J Med 2001;344:556-63

17. Effect of intravenous corticosteroids on death within 14 days in 10,008 adults with clinically significant head injury (MRC CRASH trial): randomized placebo-controlled trial. Lancet 2004;364:1321 